\title{
Review of immunohistochemical typing of endometrial carcinoma at the Lagos University Teaching Hospital
}

\author{
Olayemi Olubunmi Dawodu ${ }^{1}$, Kehinde Sharafadeen Okunade ${ }^{2}$, Adetola Daramola ${ }^{1}$, \\ Adekunbiola Aina Fehintola Banjo ${ }^{1}$
}

1. Department of Anatomic and Molecular Pathology, College of Medicine, University of Lagos.

2. Department of Obstetrics \& Gynaecology, College of Medicine, University of Lagos.

\section{Emails:}

kehindeokunade@gmail.com,sokunade@unilag.edu.ng

\begin{abstract}
Background: Categorization of endometrial carcinomas as type I and II provides useful insights into their different risk factors, pathogenesis and biologic behaviours.

Aim: To determine the immunohistochemical classifications of endometrial carcinomas in Nigerian women.

Design: A retrospective review of histopathologic slides of cases of endometrial carcinomas seen at the Lagos University Teaching Hospital (LUTH) over a 5-year period. The slides were reviewed, and the diagnoses made according to the WHO nomenclature. The classification of endometrial carcinomas into Type I and II was made by immunohistochemistry using antibodies to ER, PR, p53 and Ki-67.

Results: Eight cases of endometrial adenocarcinoma were reported accounting for $53.3 \%$ of all endometrial malignancies. Of these, only 1 case showed the classic type I immunophenotype while type II staining pattern was seen in 4 cases. The remaining 3 cases had equivocal immunophenotypes: one was p53+ but showed ER+, PR+ and high Ki-67 index; the second was p53-, ER+, PR+ but had a high Ki-67 expression; while the last was p53-, but ER-, PR- and had high Ki-67 expression.

Conclusion: Endometrial carcinomas in Nigerian women are more likely to be type II carcinomas. A reasonable proportion of the cases were equivocal thus requiring further categorization with molecular studies.
\end{abstract}

Keywords: Endometrial carcinomas, immunohistochemistry, LUTH, Nigerian.

DOI: https://dx.doi.org/10.4314/ahs.v19i3.22

Cite as: Dawodu OO, Okunade KS, Daramola A, Banjo AAF. Review of immunohistochemical typing of endometrial carcinoma at the Lagos University Teaching Hospital. Afri Health Sci. 2019;19(3): 2468-2475. bttps:/ / dx.doi.org/10.4314/abs.v19i3.22

\section{Introduction}

Carcinoma of the endometrium is the most common gynaecological malignancy in developed countries. The global incidence is 23.2 per 100,000 and it is a disease of post-menopausal women with only $25 \%$ presenting before menopause. The mean age is 60 years with a focal incidence age of between 55 and 70 years. ${ }^{1}$ The diagnosis of endometrial carcinoma when made before the age of 40 years, usually involves abnormal syndromes. ${ }^{2,3}$ Afri-

\section{Corresponding author:}

Kehinde Sharafadeen Okunade,

Department of Obstetrics \& Gynaecology,

College of Medicine, University of Lagos,

PMB 12003, Lagos, Nigeria

Phone numbers: 08034728139,08177440443

Email:kehindeokunade@gmail.com
can-American women have poorer survival at each stage of disease and are more likely to be diagnosed with late stage endometrial cancer. ${ }^{4-6}$

The risk factors for endometrial cancer include early menarche, late menopause, and nulliparity; increased total length of menstruation span, sub-fertility and repeated miscarriages usually producing an unopposed estrogen effect on the endometrium with resultant excessive proliferation. Other factors are obesity, tamoxifen use, estrogen replacement therapy (ERT), hypertension, a diet high in animal fat, prior pelvic irradiation, polycystic ovarian syndrome diabetes, family history, and past history of breast ovarian or intestinal malignancy., $3,4,8$ There have been reports of occurrence of carcinoma of the endometrium in women taking oral contraceptive agents for long periods with duration ranging from $1-4.5$ years. ${ }^{1,5,6,8-11}$

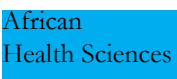

2468

(C) 2019 Dawodu et al. Licensee African Health Sciences. This is an Open Access article distributed under the terms of the Creative commons Attribution License (https://creativecommons.org/licenses/BY/4.0), which permits unrestricted use, distribution, and reproduction in any medium, provided the original work is properly cited. 
Patients with endometrial adenocarcinoma fall into two loose clusters clinicopathologically. ${ }^{9}$ This categorization as type I and II provides useful insights into their different risk factors, pathogenesis and biologic behaviors. Making the distinction between type I and II endometrial carcinoma remains a common problem in general practice. For ambiguous cases, meticulous attention to morphology and a battery of immunohistochemical studies may be required. ${ }^{12,13}$ A panel of immunohistochemical markers is often useful in difficult cases as no single marker provides excellent statistical performance. ${ }^{14}$ Type I carcinomas are typically low grade tumours, hormone dependent and usually associated with endometrial hyperplasia. They therefore occur in the setting of obesity, nulliparity, late menopause, prolonged unopposed estrogen exposure etc., and are ER+, PR+, p53- with low Ki-67 labeling index. Type II cancers occur in older individuals, are not hormone dependent, and are high grade tumours with aggressive behavior. They are ER-, PR-, P53+ and have high Ki-67 expression.

Many publications regarding the usefulness of the four markers in this panel have reported individual performance of the markers in a cohort of cases ${ }^{15-17}$ but when all four are examined together as many as $70 \%$ of endometrial tumours do not adhere strictly to the anticipated patterns, with aberrant positive or negative expression of at least one of the four markers in the panel. ${ }^{16}$ Despite this, type I endometrial cancers account for the majority of cases in Western literature; however little has been published in the African population. There is also little information on endometrial carcinoma using immunohistochemistry (IHC) staining in Nigeria as only a few publications are available in this regard. ${ }^{18-20}$ This study will therefore provide information on the distribution of endometrial carcinoma among women seen at the Lagos University Teaching Hospital using IHC staining for estrogen (ER) and progesterone receptor (PR), p53 and Ki-67 markers. This will thus enrich the current literature available in Nigeria with molecular information on endometrial carcinoma, which is fast becoming the current recommendation for choice of therapy and prognostication of the disease.

\section{Methodology}

The study was a retrospective review of histopathologic slides and request forms of cases of endometrial carcinomas recorded in the register of the Department of Anatomic and Molecular Pathology of the Lagos Uni- versity Teaching Hospital (LUTH) between January $1^{\text {st }}$, 2007 and December 31 ${ }^{\text {st }}$ 2011. The pathological reports of the cases were reviewed noting the clinical indication, age, morphological description of lesions and the histological types.

All the specimens were collected from suspected and confirmed endometrial cancer cases in the Gynaecological units of the hospital and then were fixed and transported in 10\% buffered formalin medium to the Department of Anatomic and Molecular Pathology. The slides were reviewed, and the diagnosis of endometrial carcinomas confirmed. New sections were cut from paraffin-embedded tissue blocks and stained routinely with haematoxylin and eosin. In some cases, special histochemical technique such as Ziehl-Nielsen stains are employed to demonstrate acid fast bacilli (AFB) and periodic acid Schiff (PAS) to differentiate between carcinoma and sarcoma. The World Health Organization (WHO) classification of endometrial carcinoma was used. ${ }^{9}$ Included in the study were slides obtained from all endometrial cancer samples obtained from endometrial samplings and hysterectomy samples during the period under review and excluded were slides of patients with inconclusive clinical indication or missing biodata on histopathological forms and biopsies with inadequate sampling, poor fixation and specimens lacking surface epithelium.

The classification of endometrial carcinomas into type I and type II using antibodies for estrogen receptor (ER), progesterone receptor (PR), p53 and $\mathrm{Ki}-67$ was done with immunohistochemistry. Type I stains ER +ve, PR +ve, p53 -ve and Ki-67 -ve and type II stains were ER -ve, PR -ve, p53 +ve and Ki-67 +ve. The slides were taken through decreasing grades of alcohol, then rinsed in distilled water. The antigen was retrieved from the tissue with Citrate buffer for 18-20mins in a microwave, then hydrogen peroxide was added for 10 mins in a humidity chamber. The slides were then rinsed in 2 changes of phosphate buffer solution for 3 minutes at each change. The ultraviolet block was added for $5 \mathrm{mins}$, the UV block was then tipped off from the slides. The slides were flooded with 200 microlitre of prediluted antibodies and incubated for $30 \mathrm{mins}$. They were rinsed again with 2 changes of phosphate buffer for 3 mins each time and antibody enhancer was added for 30 minutes, it was then rinsed with 2 changes of phosphate buffer. The slides were incubated with horseradish peroxidase for $30 \mathrm{mins}$ 
and rinsed with 2 changes of phosphate buffer. The already prediluted chromogen was added for 5 mins and then rinsed with 2 changes of phosphate buffer. The colour differentiation was done with haematoxylin for 2 minutes, and then finally rinsed with water. The immunohistochemistry slides were reported using corresponding control slides for each antibody as well as internal control on the individual slides.

The Epi info version 7.2 statistical software manufactured by the US Centers for Disease Control and Prevention was used for data entry and validation. Descriptive statistics were computed for all relevant data and results presented in tables and charts. Ethical approval for the study was obtained from the Health Research \& Ethics Committee of LUTH.

\section{Results}

Out of the total of 613 endometrial specimens received and processed during the review period (January 2007 and December 2011), only 15 cases of uterine malignancies were diagnosed on histology. The malignant lesions were distributed as 8 cases of endometrial adenocarcinoma, 3 cases of endometrial stromal sarcoma and 4 cases of mixed mullerian tumour (Figure 1).

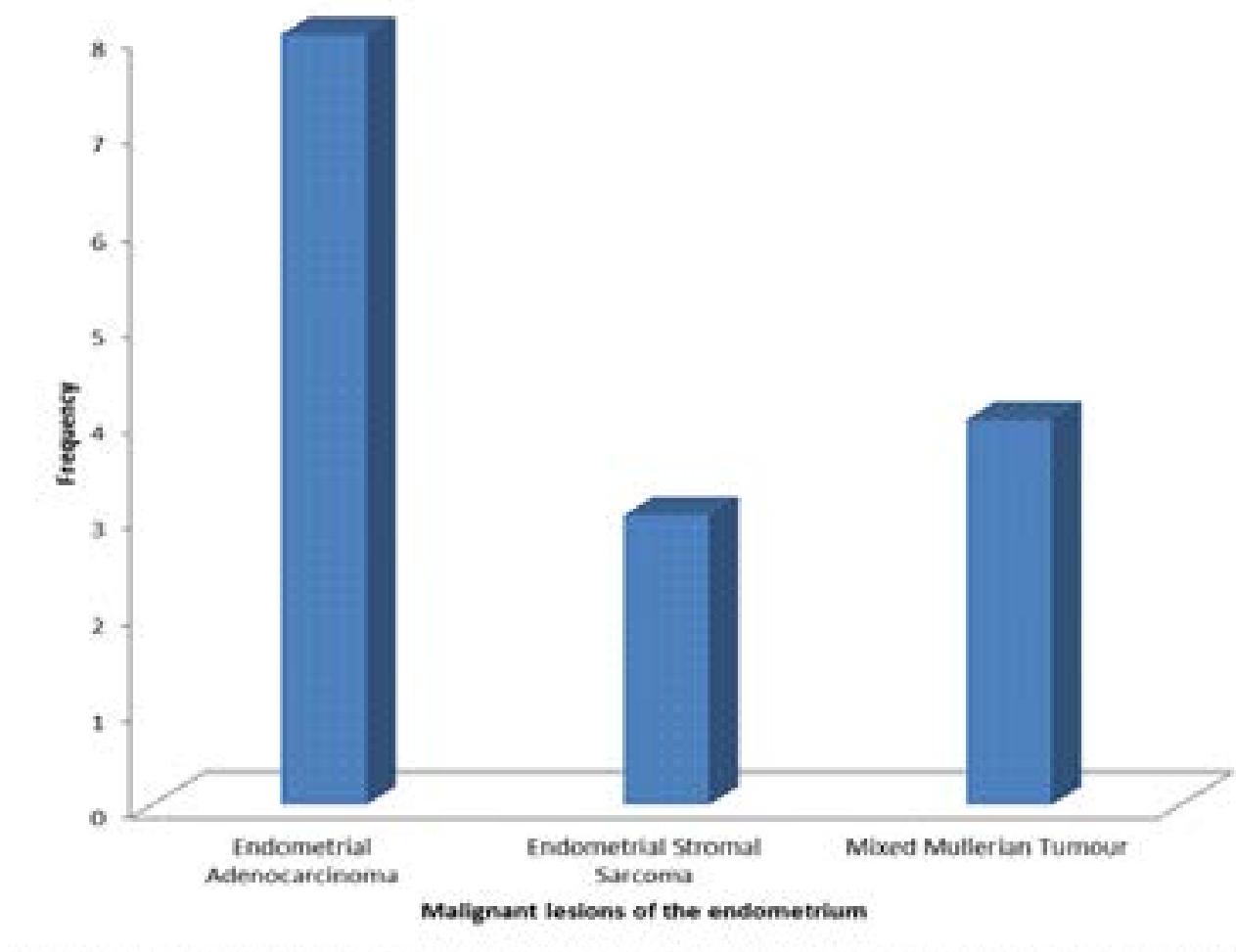

Figure 1: Distribution of malignant lesions of the endometrium in the study

All the cases of endometrial cancer recorded in the study occurred from the $5^{\text {th }}$ decade of life onward with the mean age at diagnosis being 52years. Majority (62.5\%) of the endometrial cancer specimens examined in the study were obtained from hysterectomy while the largest proportion $(62.5 \%)$ of the specimens had grade II diseases (Table 1). 
Table 1: Socio-demographic characteristics of the study participants/ specimens

\begin{tabular}{lll}
\hline Characteristics & Frequency $(\mathbf{n}=\mathbf{8})$ & Percentage (\%) \\
\hline Age (in years) & 4 & 50.0 \\
$41-50$ & 2 & 25.0 \\
$51-60$ & 1 & 12.5 \\
$61-70$ & 1 & 12.5 \\
$71-80$ & 5 & \\
\hline Type of specimen examined & & 62.5 \\
Hysterectomy & 3 & 37.5 \\
Endometrial curetting & & \\
Grade of the lesions & 1 & 12.5 \\
Grade I & 5 & 62.5 \\
Grade II & 2 & 25.0 \\
Grade III & & \\
\hline
\end{tabular}

In table 2, a panel of immunohistochemistry staining was performed on the 8 cases of endometrial carcinoma using ER, PR, p53, Ki-67 to further differentiate them into type I and type II as a baseline to determine which is more common in our environment. The only type I immunophenotype was reported in a 44-year-old woman with well differentiated endometrioid carcinoma. Type II immunohistochemistry staining pattern was seen in four cases, of which two had histologic diagnosis of high grade tumours. The three equivocal cases seen were in their fifth decade of life and had grade II tumours.

Table 2: Endometrial carcinoma typing using immunohistochemistry panel

\begin{tabular}{|c|c|c|c|c|c|c|c|}
\hline $\begin{array}{l}\text { Immuno- } \\
\text { phenotype }\end{array}$ & Age & ER & PR & Ki-67 & P53 & $\begin{array}{l}\text { Histological diagnosis } \\
\text { of endometrial tissue }\end{array}$ & Grade \\
\hline Type I & $44 \mathrm{yrs}$ & $+\mathrm{ve}$ & $+\mathrm{ve}$ & -ve & -ve & Endometrioid carcinoma & $\mathrm{I}$ \\
\hline \multirow[t]{4}{*}{ Type II } & $61 \mathrm{yrs}$ & -ve & -ve & $+\mathrm{ve}$ & $+\mathrm{ve}$ & $\begin{array}{l}\text { Endometrial } \\
\text { adenocarcinoma }\end{array}$ & III \\
\hline & $57 \mathrm{yrs}$ & -ve & -ve & $+\mathrm{ve}$ & $+\mathrm{ve}$ & $\begin{array}{l}\text { Endometrioid } \\
\text { adenocarcinoma }\end{array}$ & II \\
\hline & $55 \mathrm{yrs}$ & -ve & -ve & $+\mathrm{ve}$ & $+\mathrm{ve}$ & $\begin{array}{l}\text { Endometrial } \\
\text { adenocarcinoma }\end{array}$ & III \\
\hline & $76 y r s$ & -ve & -ve & $+\mathrm{ve}$ & $+\mathrm{ve}$ & $\begin{array}{l}\text { Endometrial } \\
\text { adenocarcinoma }\end{array}$ & II \\
\hline \multirow[t]{3}{*}{ Equivocal } & $48 \mathrm{yrs}$ & -ve & -ve & $+\mathrm{ve}$ & -ve & $\begin{array}{l}\text { Endometrial } \\
\text { adenocarcinoma }\end{array}$ & II \\
\hline & $49 \mathrm{yrs}$ & $+\mathrm{ve}$ & $+\mathrm{ve}$ & $+\mathrm{ve}$ & $+\mathrm{ve}$ & $\begin{array}{l}\text { Endometrial } \\
\text { adenocarcinoma }\end{array}$ & II \\
\hline & $41 \mathrm{yrs}$ & $+\mathrm{ve}$ & $+\mathrm{ve}$ & $+\mathrm{ve}$ & -ve & $\begin{array}{l}\text { Endometrial } \\
\text { adencarcinoma }\end{array}$ & II \\
\hline
\end{tabular}


Figure 2 showed the photomicrographs of type 1 endometrial carcinoma immunohistochemistry profile while figure 3 showed the photomicrographs of type 2 endometrial carcinoma immunohistochemistry profile.

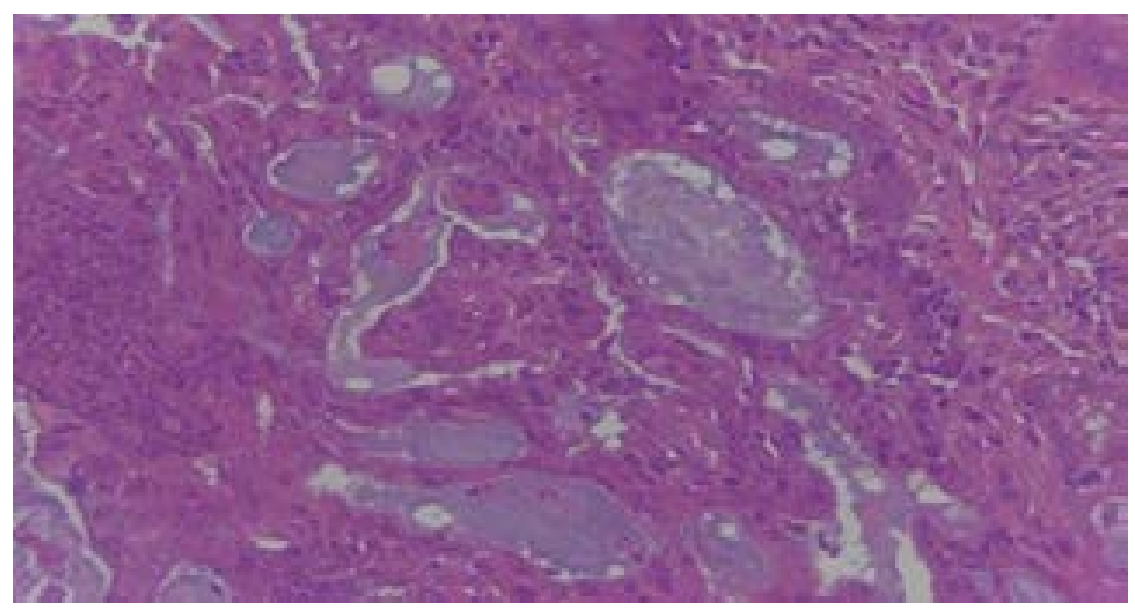

H\&E x400

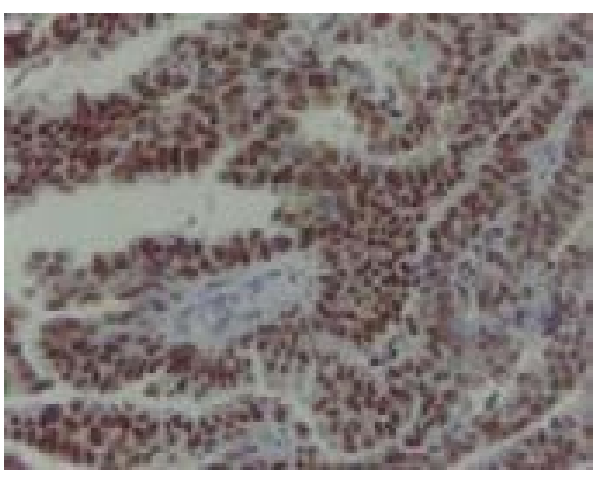

ER positive

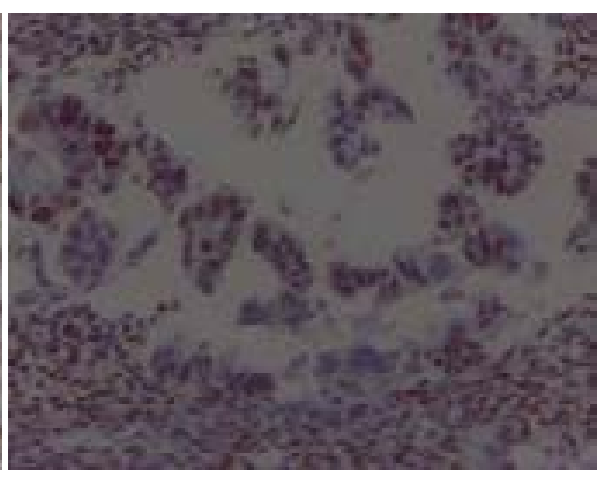

PR positive

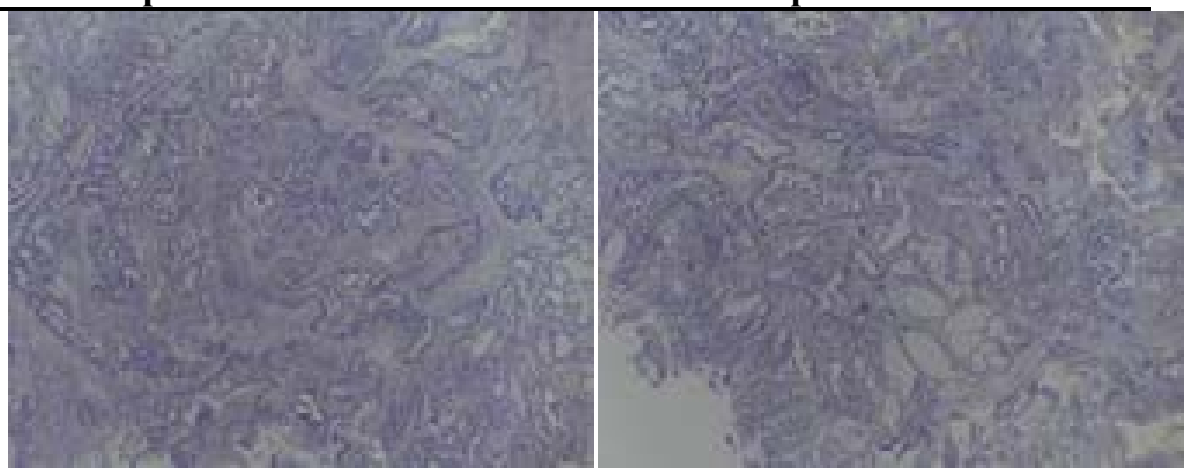

Ki67 negative

P53 negative

Image 1: Photomicrograph showing Type 1 Endometrial carcinoma immunohistochemistry profile. 


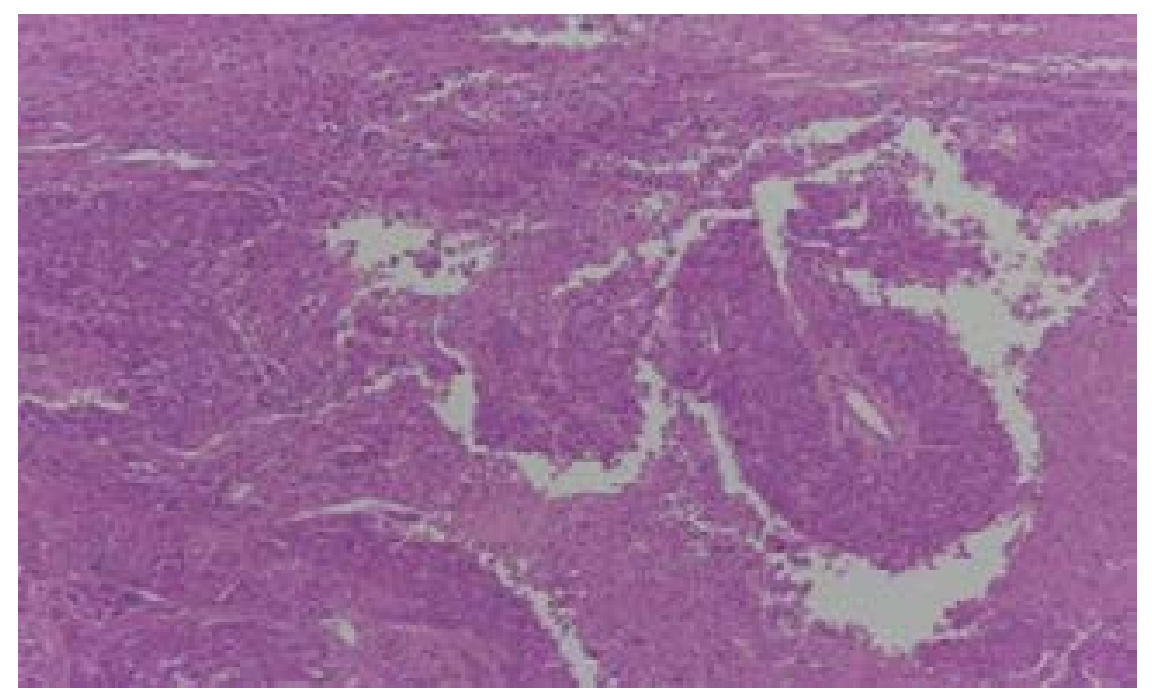

H\&E $\mathbf{x} 400$

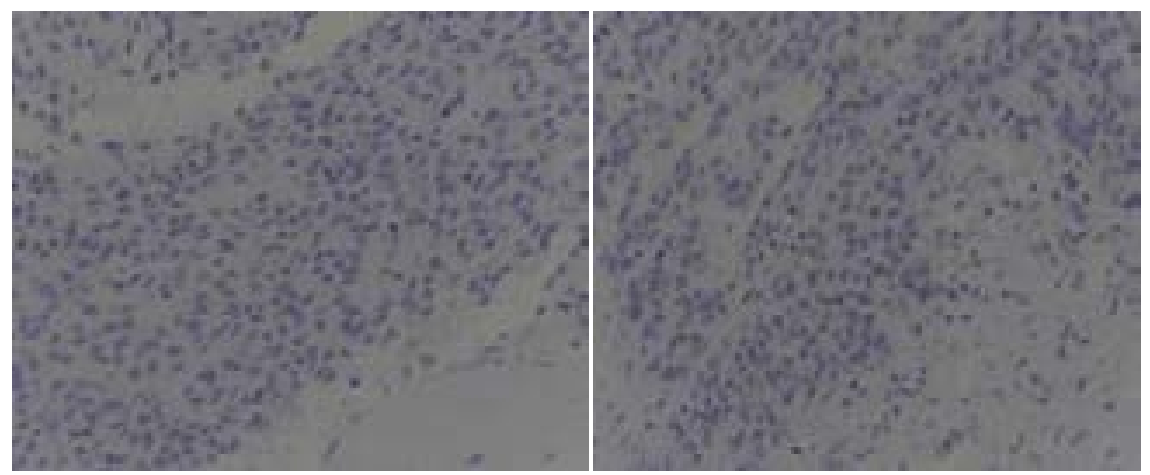

ER positive

PR positive

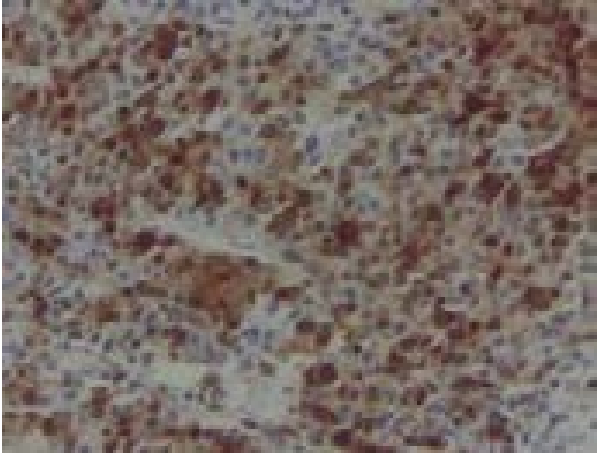

Ki67 negative

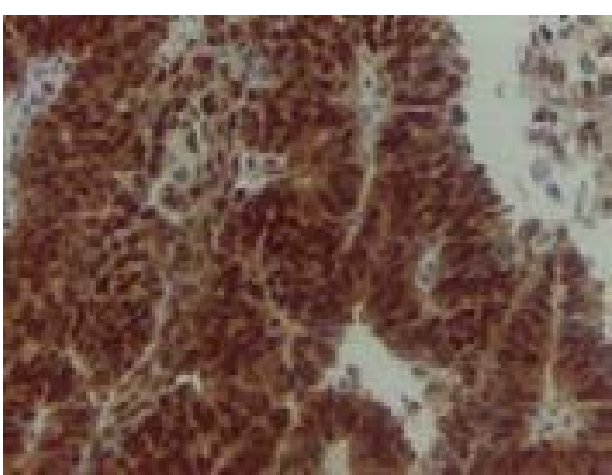

P53 negative

Image 2: Photomicrographs showing Type 2 Endometrial carcinoma immunohistochemistry profile

\section{Discussion}

In this study, there were 8 cases of endometrial adenocarcinoma, 3 cases of endometrial stromal sarcoma and 4 cases of mixed mullerian tumour accounting for $12.4 \%$ of neoplastic lesions and 3.2\% of all biopsies. This is in keeping with reports from Calabar, ${ }^{19}$ Kaduna $^{21}$ Ibadan $^{22}$ and Pakistan ${ }^{23}$ where adenocarcinoma also accounted for majority of endometrial malignancies seen. This study 
also showed that most patients with the disease were in their fifth decade of life and this is similar to the findings by Galadanci et al in Kano, ${ }^{24}$ Mandong in Jos, ${ }^{20}$ but is in contrast to the findings by Ogunbode et al in Ibadan. ${ }^{18}$

The use of immunohistochemistry to type endometrial adenocarcinoma showed that only one of the eight patients, a 44-year-old lady with a previous family history of endometrial and breast cancer in her mother and sister had a type I profile. The association of Lynch syndrome appeared probable in this case, ${ }^{25}$ however further tests to assess for microsatellite instability and epigenetic alterations are unavailable. The patient and family members need appropriate surveillance measures due to the increased risk of developing other cancers such as cancer of the colon and ovary. Although a previous study has shown Lynch-syndrome associated endometrial carcinoma presenting in women older than 50 -years who later developed a second cancer within a median time of 11 years. ${ }^{26}$ The four patients with type II immunophenotype had an age range of 57 to 76 years and had moderately differentiated to poorly differentiated adenocarcinomas. The type II cases are usually associated with high grade tumours as seen in the 61-year-old woman with diffuse nuclear atypia and a greater than $50 \%$ solid growth in this study. ${ }^{27}$ It thus appears from this study that we have more type II tumours based on immunohistochemistry, and thesare usually high-grade neoplasms with worse prognosis. $^{27}$

As a previous study has shown that as many as $70 \%$ of endometrial carcinoma do not adhere strictly to the anticipated patterns of IHC stainings, ${ }^{16}$ our study only reported three equivocal cases (less than 40\%) whose staining patterns did not fall into any of the two classes of endometrial cancer. The classification of endometrial carcinoma into type I and type II though helpful is rigid and some tumours are grouped into the same category but have different clinical, immunohistochemical and molecular features. ${ }^{28}$ There is a need, therefore, to always emphasize that the diagnosis of endometrial carcinoma is largely morphologic as IHC use has lots of overlaps in this entity. It is therefore pertinent to hold morphologic diagnosis as the gold standard while IHC can serve as adjuncts. However, a major limitation of this study is the very few endometrial cancer cases analyzed, perhaps a more robust multi-center study with larger sample size if done in the nearest future, might reveal a change in the distribution of endometrial cancer cases.

\section{Conclusion}

Endometrial carcinomas in Nigerian women are more likely to be type II carcinomas. A reasonable proportion of cases in this population are equivocal requiring further categorization with molecular studies. Even though, the use of immunohistochemistry helps to provide accurate diagnosis in endometrial carcinomas which will thus aid the in prognostication and choice of therapy, our study suggests that there may be no need for such expensive studies in our environment as histological grade corresponds well with the immunohistochemistry typing as we have also seen in most resource-poor settings. Thus, immunohistochemistry should only be reserved for cases with ambiguous or discordant histological diagnosis.

\section{Acknowledgements}

The authors appreciate the assistance given to us by the staff of the Departments of Anatomic \& Molecular Pathology, Obstetrics \& Gynaecology and Medical record of Lagos University Teaching Hospital (LUTH). The work reported in this publication was partly supported by the Fogarty International Center and National Institute of Mental Health, of the National Institutes of Health under Award Number D43TW010543. The content is solely the responsibility of the authors and does not necessarily represent the official views of the National Institutes of Health.

\section{Conflict of interests}

The authors declare no conflict of interests in carrying out this study.

\section{References}

1. Coleman MP, Esteve J, Damiecki P, Arsian A, Renard $\mathrm{H}$. Trends in cancer incidence and mortality IARC scient. Publ No 121: International Agency for Research 1993.

2. Anderson Barrie. Diagnosis and Staging of Endometrial Carcinoma. Clinical Obstetrics and Gynae 1982; 25: 75-80

3. Creasman W, Morrow C, Bundy B, Kurman RJ, Heller P, Homesley HD et al. Surgical pathologic spread patterns of endometrial cancer. A Gynecologic oncology group study cancer 1987; 60: 2035-2042.

4. Delgish L, Cohen CJ. Histological correlates and vir- 
ulence implications of endometrial carcinoma associated with adenomatous hyperplasia. Am. Obstet. Gynae. 1985; 56: 1452-1455.

5. Brinton LA, Hover RN. Epidemiology of Gynaecologic cancer in: Principles and Practice of Gynaecologic oncology. WS Hoskins, CA Berez and RC Young (Eds). Philadelphia Lippincot Raven, $2^{\text {nd }}$ ed, 1997: 3-29.

6. Barakat RR. Endometrial cancer and Tamoxifen. Clin Obstet Gynaecol 1996; 39: 629-40.

7. Chen LM, McGonigle KF, Bereck JS. Endometrial Cancer. Recent developments in evaluation and treatment oncology. 1999; 13: 1665 - 1682.

8. Parazzini F, La Vecchia C, Bocciolone L, Franceschi S. The epidemiology of endometrial cancer Gynae Oncol. 1991; 41:1-16.

9. Scully RE, Bonfiglio JA, Kurman RV, Silverberg SG, Wilkinson EJ. Histological Typing of female genital tract tumours. In: World Health Organization. International Histological classification of Tumours. New York: Springer-Verlag 2001; 92: 2578 - 2584. PubMed

10. Cohen CJ, Deppe G. Endometrial carcinoma and oral contraceptive agents, Obstet and Gynaecol. 1977; 49: 390392.

11. Sherman ME, Sturgeon S, Bruton LA Potischman N, Kurman RJ, Berman ML, et al. Risk factors and hormone levels in patients with SENAS and endometrial uterine carcinoma. Mod. Path. 1997; 10: 963-968. PubMed

12. Bartosch C. Manuel Lopes J, Oliva E. Endometrial carcinomas: a review emphasizing overlapping and distinctive morphological and immunohistochemical features. Adv Anat Pathol. 2011;18(6):415-437.

13. McCluggage WG. Immunohistochemical and functional biomarkers of value in female genital tract lesions. Int J Gynecol Pathol. 2006;25(2):101-120.

14. Wei J-J, Paintal A, Keh P. Histologic and Immunohistochemical Analyses of Endometrial Carcinomas: Experiences from Endometrial Biopsies in 358 Consultation Cases. Arch Pathol Lab Med. 2013;137:1574-1583. PubMed 15. McCluggage WG. A critical appraisal of the value of immunohistochemistry in diagnosis of uterine neoplasms. Adv Anat Pathol 2004; 11(3): 162-71.

16. Djordjevic B, Westin S, Broaddus RR. Application of Immunohistochemistry and Molecular Diagnostics to Clinically Relevant Problems in Endometrial Cancer. Surg Pathol Clin. 2012; 5(4): 859-878. PubMed
17. McCluggage WG. Recent advances in immunohistochemistry in gynaecological pathology. Histopathology 2002; 40(4): 309-26.

18. Ogunbode DE, Aimakhu VE. Carcinoma of the Corpus Uteri in Ibadan, Nigeria, East African Med. Journal 1974; 51: $647-652$.

19. Ekanem IA, Ekanem AD: Endometrial pathology associated with infertility among Nigerian women, Niger Postgrad Med J. 2006; 13(4), 344-347

20. Mandong BM, Ujah IAO. A ten-year Review of Gynaecological malignancies in Jos, University Teaching Hospital, Jos. Nigeria, Sabel Medical Journal 2003; 6: 49-52. PubMed

21. Mohammed A, Ahmed SA, Oluwole OP, Avidime S. Malignant Tumour of the Female Genital Tract in Zaria, Nigeria- Analysis of 513 cases. Annals of African Medicine. 2006: 5(2): 93-96.

22. Ogunbiyi JO, Omigbodun AO. Malignant Tumours of the Corpus Uteri in Nigerian Women, Afr J Reprod Health 1999; 3(1): 81-87. PubMed

23. Jamal S, Mamoon N, Mushtaq S, Luqman M, Moghal S. The Pattern of Gynaecological Malignancies in 968 Cases from Pakistan; Ann Saudi Med 2006; 26 (5): 382384. PubMed

24. Galadanci HS, Mohammed AZ, Uzoho CC, Jido TA, Ochicha O. Gynaecological Malignancies seen in a Tertiary Health Facility in Kano, Northern Nigeria. Trop J. Obstet Gynaecol 2003; 20: 105-108.

25. Lynch HJ, Lynch J, Conway J, Watson P, Coleman RL, Familial aggregation of carcinoma of the endometrium. Am J of Obstet and gynae 1994; 24-27.

26. Lu KH, Dinh M, Kohlmann W, Watson P, Green J, Syngal S, et al. Gynecologic Cancer a "Sentinel Cancer" for Women with Hereditary Nonpolyposis Colorectal Cancer Syndrome. Obstet Gynecol 2005; 105; 569-574.

27. Hendrickson MR, Longacre TA, Kempson RL. The uterine corpus. In Mills SE, Carter D, Greenson J. K, Oberman HA, Reuter VE, Stoler MH editor Sternberg's Diagnostic surgical pathology. 4th ed. Vol. 3: Philadelphia Lippincott. Williams; 2004: 2453- 2588.

28. Catasus L, Gallardo A, Cuatrecasas M, Prat J. PIK3CA Mutations in the Kinase Domain (exon 20) of uterine endometrial adenocarcinoma are associated with adverse prognostic parameters. Mod. Pathol. 2008; 131-139. 